\title{
Visualization of interference pictures of 3D scenes including optically isotropic transparent objects
}

\author{
V.A. Debelov ${ }^{1}$, L.F. Vasilieva ${ }^{2}$ \\ Institute of Computational Mathematics and Mathematical Geophysics SB RAS \\ 1 ORCID: 0000-0002-7577-4700, debelov@oapmg.sscc.ru \\ 2 ORCID: 00o0-0001-7278-8800, vaslud@gmail.com
}

\begin{abstract}
To date, only few papers are available on the calculation of interference pictures, even for scenes with objects specified by optically isotropic materials. Major attention has been given only to some questions arising in the development of renderers. In this paper, we propose some solutions to the following problems of rendering: a) representation of a ray in rendering, that is, information about the ray which allows taking into account interference in the calculations; b) expansion of the concept of scene object material; and c) estimation of ray intensity on an image surface. The main purpose is to describe the peculiarities of spectral rendering when calculating interference effects in scenes with optically isotropic objects to be taken into account in the development of corresponding renderers, rather than the development of any specific renderer.

As an algorithm for interference calculation in an isotropic medium, we consider a direct simulation: tracing by rays of linear polarized light from a source to an image plane.

The main point of the approach is that no special interfering objects such as thin films, soap bubbles, etc. are used in a scene. Images are calculated with a spectral representation of light and materials of the scene objects, but not on the basis of an RGB model. Interference is calculated at a scene point corresponding to a pixel of the image plane where coherent rays may come along unpredictable trajectories, in particular, along trajectories passing through some known interfering objects.
\end{abstract}

Keywords: interference, linear polarization, optically isotropic transparent objects, photorealistic spectral rendering, tracing of linear polarized rays, optical path, ray intensity estimate in a pixel.

\section{Introduction}

Scientific visualization is finding increasing use in various fields of research. The range of problems where visualization is important is very wide: from physical processes based on physically justified mathematical models [1] to algorithm debugging [2]. Scientific visualization is based on many achievements in virtual and augmented reality systems. Any new functionality of virtual reality systems finds immediate use in scientific visualization, and some of its new functions are initiated by the requirements of scientific visualization. Owing to the widespread use of virtual and augmented reality systems, there are opportunities to have more realistic versions of computer-synthesized pictures and more realistic details. Visualization in such systems is performed by rendering programs which calculate images based on computer descriptions of virtual environments or scenes. Therefore, the virtual reality systems can employ only the capabilities provided by achievements in photorealistic computer graphics.

The various fields of computer graphics mutually enrich each other; for instance, a scientific visualization of tsunami and earthquake maps based on an approach to light interference visualization was proposed in [3]. 
One of the main problems of photorealistic computer graphics is to obtain (calculate, render) images of virtual computer $3 \mathrm{D}$ scenes by a virtual camera [4]. Here a virtual scene is a computer representation or a model of a real scene with the degree of detail, geometry, and optical materials for which the renderer is designed. Also, a virtual camera is a computer model of an image-recording device: a camera, a scanner, or a human eye. A camera is an integral part of any renderer construction. If a renderer construction does not take into account some peculiarities of the interaction of light with objects, the calculated image will not show them. Photorealistic computer graphics (as indicated in the name) is primarily used to calculate images of virtual scenes which are comparable to photographs of real analogs of these scenes. This is done by fully taking into account the details of physical laws of interaction of light with the material of the scene objects. Such details include interference, which often takes place in real objects, for instance, in iridescent spots of petrol on puddles [5].

The phenomenon of interference has not been given sufficient attention in computer graphics for the following reason: only coherent light rays can interfere. In our opinion, in the ordinary $3 \mathrm{D}$ scenes the light arriving at a scene point in different ways is, as a rule, incoherent. According to paper [6], if the illumination of an object of observation is not due to direct sunlight, but due to light scattered by surrounding objects or clouds, the individual points of these objects can be considered sources of incoherent waves (the size of their coherence domain is $0,06 \mathrm{~mm}$ ). Thus, it has turned out that only calculations of energy for individual rays are sufficient, without taking into account the phase of electromagnetic oscillations (the wave nature of light). At the scene point being investigated only the ray energies are added, and no phase shifts of the light waves coming to the scene point with different rays must be taken into account.

There are more complicated manifestations of interference, namely, interference pictures of anisotropic crystals $[7,8]$, which can be obtained by a conventional camera even in a very simple scene with a transparent anisotropic mineral (for instance, calcite or quartz) between the polarizers in a polariscope [9]. In the present paper, scenes with optically anisotropic objects are not considered.

Here we consider a direct simulation of the propagation of linear polarized light rays in a scene with optically isotropic objects and media, that is, a calculation of the propagation of light rays from a source to an image plane. An important characteristic of the algorithm is that the tracing is performed by linear polarized rays of "zero thickness" [10], that is, by rays used in traditional renderers (in contrast, for instance, to Gaussian solid beams [10]). Some issues are considered with a description of computer experiments in which images are calculated by taking into account the interference of simplest scenes containing transparent optically isotropic objects. Special attention is given to some peculiarities of the calculation of interference effects to be taken into account in the development of corresponding renderers.

In the present paper, diffraction is not considered. It plays an important role, but requires time-consuming calculations, although this phenomenon is not always visible in ordinary scenes.

Few works have been published on the calculation of interference pictures, even for scenes with objects specified by optically isotropic materials. Major attention has been given only to some questions arising in the development of renderers. In the present paper, we propose some solutions to the following questions:

- Representation of a ray in rendering, that is, information about the ray which allows taking into account interference in the calculations.

- Expansion of the concept of scene object material.

- Ray intensity estimation on an image surface.

An attempt is made to gather the basic technical information and present it in sufficient detail. The goal of the authors is to describe the peculiarities of calculation of the interfer- 
ence effects to be taken into account in the development of appropriate renderers, but not the development of any specific renderer.

In Section 2, some of the available papers on visualization are considered. Section 3 provides a brief description of light polarization and the calculation of intensity in the case of two-ray and multi-ray interference. Some proposals are made on modifying the concept of object material and the information load of a tracing ray to provide flexible programming of scene rendering in the presence of interference. Section 4 is devoted to numerical experiments based on classical scenes: Young's experiment and amplitude division [5]. In Section 5, an approach is proposed to the resulting intensity calculation in an image plane pixel using the wave nature of light.

\section{Previous work}

Interference pictures have been much studied in the scientific, educational, and technical literature devoted to optics (see, for instance, $[5-7,11]$, and many others). All such studies do not consider computer graphics and describe the physics of some observed pictures depending on the material of the sample and its parameters. Interference pictures are a tool for identifying the material (mineral), evaluating the quality of a product (for instance, sugar), etc. Among the most well-studied objects are well-polished plane-parallel plates and wedges. Optical devices, such as interferometers [5, 11, 12] and others, have been studied in detail, since they are of great practical importance. Thin films (such as spots of petrol on water and paint coatings) have also been studied in detail. The calculations are mainly made in a small neighborhood of the interaction of a ray with a plate or a thin film. The way in which an interference picture is obtained is usually not clearly formalized. It is only stated that the interference picture is in the focal plane of a lens or in an eye at infinity (see $[5,6]$, and others).

The available programs of photorealistic rendering allow obtaining images for scenes in which the optical properties called materials (coefficients of reflection, refraction, etc.) of objects and sources are specified with spectra in a visible wavelength range from 380 to $780 \mathrm{~nm}$. The phenomenon of optical dispersion, that is, the phenomenon in which the refraction angle of a light ray depends on the wavelength (the refractive index of the medium), can be taken into account by using so-called spectral rendering [4, 13]. Hence, Newton's prism experiment on white light decomposition into its spectrum can be programmed. This has been demonstrated by many modern renderers (for instance, Maxwell render [14]). Spectral rendering has made it possible to calculate physically correct images of diamonds, rhinestones, glass objects, etc.

In photorealistic computer graphics, attention was naturally given to the phenomenon of interference, which often manifests itself in everyday life. Let us consider the most wellknown approaches.

First of all, there appeared papers in which interference in thin films was calculated on the basis of analytical solutions, mathematical models, and formulas available in the physical literature. One of the first works of this type was presented by Diaz [15]. A series of papers is devoted to simulation of interference on thin films (see, for instance, the paper of Glassner [16]). With given parameters, the required image for a scene consisting of two or three soap bubbles was calculated by corresponding formulas. Unfortunately, optical dispersion was not taken into account, since the same refractive index was used for all wavelengths. In fact, in this case computer graphics was used on the level of a pixel, line, or symbol. Various specialized systems or simulators can be constructed in this way, but this is not photorealistic computer graphics proper.

In relation to computer graphics and interference calculation, one should mention paper [17], in which it was proposed to render by tracing polarized rays and take into account the Fresnel decomposition [5, 6] when calculating ray-surface interactions. A special scene object (a thin film, a varnish or paint layer) was identified. The authors proposed a para- 
metrized geometrical and optical model of this object with interference effects to calculate an analog of the function of reflection from the object depending on the angle of the incident ray with the normal. All interactions with such objects were made by using this reflection function. In their numerical experiments the authors used the same refractive index for the material of the interfering layer; that is, optical dispersion was neglected.

The methods of computer graphics were most clearly presented and implemented in papers $[18,19]$, in which images with interference in thin films were calculated. In their subsequent paper [20] the authors also used a polarized light decomposition into parallel and perpendicular components and the Fresnel formulas.

Thus, according to these papers, the modification of a traditional renderer (as a rule, an RGB renderer) is, in simple form, as follows: a) a ray of a typical form (for example, in the form of an RGB triple) falls on an interfering layer or an object; $b$ ) the energy transferred by the ray is converted to spectral form; c) interference is calculated inside the object: the outgoing ray is calculated in spectral form; d) the outgoing ray is transformed back from spectral form to RGB; e) the RGB ray continues to move in the scene. At the second stage (b), analytical solutions for thin films [18-20] or special reflection functions are used [17].

Paper [20] describes a method for embedding interfering objects (here films) into Pixar's RenderMan [21], a renderer which is based on an RGB light ray representation.

Many papers are mainly devoted to simulating the geometry of soap bubbles and speeding up the calculation of pictures, but pay less attention to exact calculation of interference. Most likely, the purpose of such works is to obtain rainbow colors close to natural ones (for instance, textures, as in [22]).

We have discussed some representative works on the phenomenon of interference. For more comprehensive lists of literature the reader is referred to reviews in these papers.

Note the following typical peculiarities of these works:

- As a rule, a traditional representation of a ray is used, except when it is "inside" an interfering scene object. That is, in the scene itself the ray has only information about the intensities of the RGB color components.

- It is not clear how the optical characteristics of an interfering object are specified, that is, no specifications of the material are given.

- In the above-mentioned works nothing is said about the camera or the image plane. As regards the rendering of the entire scene, a ray is transmitted in a specific format to the module that traces rays inside the object, perhaps for calculating the interference, and receives the rays in the same format on the way out.

- Optical dispersion is often neglected in the numerical experiments.

- In most cases, obtaining of rainbow colors is guaranteed, which makes the image realistic but not physically correct.

There is an absolutely different approach based on physical optics, that is, taking into account the wave nature of light. In paper [10], it is proposed to perform tracing of not traditional rays of zero thickness, but a complex tracing based on Gaussian spherical waves or Gaussian beams. Only paraxial rays are used, which is often sufficient in the development of models of microscopes, lenses, etc. To date, many rendering algorithms have been proposed (see [4]) which, instead of a mathematical beam of zero thickness, use solid objects for tracing: a cone, a beam of rays, or a pencil (a beam with a central ray). None of them have been universally adopted for realistic and fast rendering of complex scenes. The present authors think that it is not realistic to construct an entire renderer on the basis of Gaussian beams even in the near future, despite the fast development of computer technology: It will be expensive and take a long time. However, it is quite feasible for optical systems, when a small number of elements is centered along a single axis.

In the present paper, an approach to modernizing the existing photorealistic spectral rendering is proposed for a physically correct simulation of the phenomenon of light interference. In general, interference cannot be taken into account without considering light polarization. Therefore, we will have to modernize the representation of a ray of light by intro- 
ducing parameters of the state of its polarization. On the other hand, a more accurate specification of the object material as a set of its optical properties is needed.

An extensive review of works on rendering by a ray of polarized light and rendering using a polarized ray of optically anisotropic transparent materials can be found in [23]. Algorithms of interaction of linear polarized light with transparent optically isotropic and anisotropic objects are considered in [2, 24-27].

Various aspects of rendering that can be used to calculate interference pictures are covered in a number of conference talks: on the development of a mathematical model of a polarized ray $[22,28,29]$; and on the interaction of polarized light with isotropic lenses [30].

Without polarization, only Young's experiment on division of wave front [5] or similar experiments can be simulated in a physically correct way, when light upon its passage from a source to a screen (eye) is not reflected and refracted on the scene surfaces (since they are absent). Hence, in this case light is not polarized even partially.

Reliability and physical correctness of the algorithms. The algorithms for visualization of interference effects are mostly based on a correct application of physical laws and formulas. For the photorealistic rendering algorithms in computer graphics, an approach to verifying the reliability and physical correctness of the rendering algorithms was proposed in the CornellBox project [31]. Two "identical" scenes were prepared: a very simple real scene consisting of several cubes having diffuse colors and a virtual computer scene. A photo of the scene was compared pixel by pixel with an image synthesized by a virtual camera based on a calculation using a radiosity method. It was demonstrated in papers $[26,32]$ that the pixel-by-pixel comparison of a photograph and results of an algorithm for interaction of rays of light with transparent anisotropic objects, using a convex calcite hexagon as an example, is reliable. Note that (visual) reliability can be confirmed by an expert when a photo and a synthesized image are evaluated by an observer (see, for instance, paper [25] in which birefringence is simulated).

In the above algorithms for the simulation and calculation of interference, no similar tests have been made, and it is not clear whether the calculated images are obtained due to interference or optical dispersion. The authors of paper [33] note that diffraction (CD discs), interference (oil spots, films), dispersion (light decomposition by a prism), scattering (rainbow), that is, four different optical mechanisms can be sources of rainbow colors. Moreover, the observer might think that the image under inspection was calculated without possible interference effects; for instance, this might be a result of calculation of caustics, as in [34]. In other words, before demonstrating a synthesized image to the reader, he should be prepared by describing the details of the scene and eliminating the influence of optical dispersion and other phenomena. In our opinion, the most realistic and reliable demonstrations of interference are the results of calculations of images for scenes with monochrome sources.

\section{Light polarization and interference}

Note that, in contrast to geometric optics, in wave optics the term "ray" is used for brevity, to denote the direction of propagation of light energy. In what follows, this term will be used without quotation marks.

\subsection{Polarization}

Light ray polarization is a characteristic that describes the behavior of the electric field vector in a plane perpendicular to the direction of ray propagation. Polarization can be linear, circular or, in the general case, elliptical [5]. Every individual electromagnetic wave is completely polarized. A ray means a quasi-monochrome light ray as a totality of waves propagating along a straight line and having the same wavelength [5]. It can be fully polarized, partially polarized, or unpolarized, depending on the degree of correlation of oscillations of individual waves in the ray. 
The following peculiarities are important for the calculations (according to [5]):

- A wave of natural (unpolarized) light of intensity $I$ is equivalent to two independent linear polarized waves whose intensity is equal to $I / 2$ and with electric vectors oscillating in two mutually perpendicular planes that are normal to the direction of propagation.

- Partially polarized light can be represented as the sum of fully unpolarized and fully polarized parts that are independent of each other.

- Two waves that are polarized in mutually perpendicular planes do not interfere.

- Rays with circular and elliptical polarization can be represented as a combination of linear polarized rays. Then, without loss of generality, calculations with fully polarized rays (and only with linear polarized rays) can be made.

There are several mathematical constructions (approaches) for describing and calculating the state of polarization: Jones vectors and Jones matrices, Stokes vectors and Mueller matrices, coherence matrices and their modifiers (see details in $[5,26,35,36]$ ). In papers [2, $26,27]$, polarization was successfully represented on the basis of coherence matrices. This representation makes it possible to describe natural (unpolarized), partially polarized, and fully polarized light, which was sufficient for the problems under consideration. Coherence matrices (like the other representations) do not keep the phase of an electromagnetic oscillation or the optical path of a ray. Therefore, none of the representations of polarization is preferable to the others.

Note that in any representation of polarization, when a ray is incident on a surface, there are clear procedures of calculating the parameters of reflected and refracted rays.

\subsection{Spectral rendering}

Assume that the renderer to be modified calculates images in the spectral rendering mode $[4,13]$. In the present paper, it does not matter what kind of ray tracing is used in the renderer for calculations: forward, reverse, recursive of the Whitted type [37], with lightmaps or with photon maps. In any case, energy is collected starting from the source. However, when maps are used, they should be considered as secondary sources along with the original ones, since they do not contain any information on coherence, or maps should also be modernized.

Light is a totality of waves from some (typically continuous) interval of electromagnetic wavelengths and is represented by a spectrum $S_{c}(\lambda)$ which is, in fact, a curve. There are cases when the spectrum curve has clear peaks; often they are considered separately. In spectral rendering, first a discretization is performed and a discrete spectrum is formed that approximates, with the needed accuracy, the curve $S_{c}(\lambda)$ as a set of $k$ samples $S d=\left\{\left\langle w_{1}, A_{1}\right\rangle, \ldots,\left\langle w_{k}, A_{k}\right\rangle\right\}$. Here $A_{i}$ is the amplitude of oscillations of the electric component for a wave of length $w_{i}$. The same set includes peaks, if any. This paper considers objects, which do not change the length of a light wave when interacting with it, like the vast majority of real-world objects. Of course, some materials, for instance, fluorescent ones, are not considered. For these, it will be necessary to refine the mathematical model.

In what follows, all formulas (unless otherwise specified) are considered for monochromatic light with wavelength in vacuum $\lambda$.

The entire process of calculation is performed for each wavelength from the set $S d$ separately, thus forming $k$ monochrome images. At the end of the process, the resulting image is formed on the basis of individual monochrome ones - the stage of color conversion from the spectrum to RGB (tone reproduction) [13, 38]. 


\subsection{Coherence}

Only coherent rays can interfere. The intensity at the point where these rays meet in the scene (on the image plane) is affected by the path difference (the difference of the optical paths). Following [5], it is assumed here that rays are coherent if they were initially emitted by a point (a small neighborhood) of any of the scene sources, and then reached this point in different ways.

In paper [39], some methods for obtaining coherent waves to perform real experiments are presented. Specifically, there are two ways to obtain waves for implementing interference in optics: 1) division of wave amplitude, and 2) division of wave front. In the numerical experiments, these methods will be considered on the basis of some classical experiments.

It is noted in papers $[5,6]$ that there is no interference picture if the path difference exceeds the coherence length. If we observe interference pictures, in such a real scene this length is not exceeded. Nevertheless, this rule should not be neglected, but the user will have an opportunity to control the parameter of coherence length - which is specific for each source.

For greater flexibility in the development of virtual $3 \mathrm{D}$ scenes, we propose to control ray coherence in the following way:

- Each ray has an attribute of optical path and an attribute of coherence. The latter defines a unique point (neighborhood) of the source that generated this ray. It can be numerical or mnemonic. If two rays have the same values of the attribute of coherence (source), they are coherent. Let the zero value mean that coherence is not defined, that is, the ray is not coherent with any other ray.

- Each source has an attribute of coherence length. If a ray generated by this source has passed more than a specified value, it will be "incoherent" in its further propagation in the scene for all other rays, that is, the attribute of coherence is set to zero.

- If a ray is refracted or reflected at its interaction with the scene surface, the user can control the coherence of generated (reflected, refracted) rays, both at the same time or each separately: a) they inherit the value of the attribute; b) lose coherence. For each scene surface, specific rules can be set. This means that the number of attributes in the concept of object material should be increased by adding the above-mentioned data control tools associated with the ray.

Note: if a source originally generated unpolarized light, two rays are generated instead of one (see above), and each of them has its own unique attribute of coherence, since they are independent.

\subsection{Interference of two coherent rays}

Let two waves emitted by a single source come to a scene point $P$, and let them be linear polarized and coherent. Their electric vectors are $\mathbf{E}_{1}$ and $\mathbf{E}_{2}$, respectively. These waves can be written as $\boldsymbol{E}=\boldsymbol{E}_{0} e^{i \phi}$, where $\phi$ is the phase, and $\boldsymbol{E}_{0}$ is the vector amplitude. The electrical component of an electromagnetic wave is important for photography, since the incoming intensity is proportional to the squared amplitude of this wave. Therefore, we will consider only this component, $I=c \cdot \boldsymbol{E}_{0}^{2}$, where $c$ is a coefficient. In what follows, we will not distinguish between the intensity and the squared amplitude, since this will not greatly change the description and calculation algorithms. The resulting electric field [5] at the scene point under consideration is the vector

Hence,

$$
E_{\Sigma}=E_{1}+E_{2}
$$

$$
\boldsymbol{E}_{\Sigma}^{2}=\boldsymbol{E}_{1}^{2}+\boldsymbol{E}_{2}^{2}+2 \boldsymbol{E}_{1} \boldsymbol{E}_{2}
$$

The total intensity $I$ is expressed in terms of the intensities of both waves as 
where the last term is interference.

$$
I=I_{1}+I_{2}+J_{12}
$$

In the literature (see $[5,11$, and others], the focus is first on the most important practical case when both waves propagate almost along the same straight line or reach the point $P$ of interest from close directions. In paper [17], their polarization planes are assumed to coincide. In these conditions the intensity at point $P$ is found by the formula

$$
I=I_{1}+I_{2}+2 \sqrt{I_{1} I_{2}} \cos \delta
$$

where $\delta$ is the phase difference of the two electric fields at $P$. For simplicity, we assume that both rays have left the source with zero phase. Let a ray (more precisely, a path) pass from the source to the point a distance of length $L_{1}$ in a medium with a refractive index $n_{1}$ ,$\ldots$, and a distance of length $L_{k}$ in a medium with refractive index $n_{k}$. Then the optical path of the ray is calculated by the formula $s=\sum_{i=1}^{k} n_{i} L_{i}$, and the phase changed by $\phi=2 \pi s / \lambda$. The two rays have passed optical paths $s_{1}$ and $s_{2}$. Then the phase difference due to the difference in the optical paths is $\delta=\frac{2 \pi}{\lambda}\left(s_{1}-s_{2}\right)$.

\subsection{Interference of several coherent rays}

Consider the case when more than two rays meet at a point. Then we use the initial formula (1) to determine the resulting value of the electric field vector. Rays converging at a point may represent some number $q$ of groups of mutually coherent rays. Then the resulting field may be represented as the sum $q$ of independent (mutually incoherent) fields:

and the sought-for intensity

$$
\boldsymbol{E}_{\Sigma}=\boldsymbol{E}_{1}+\boldsymbol{E}_{2}+\cdots+\boldsymbol{E}_{q}
$$

or

$$
\boldsymbol{E}_{\Sigma}^{2}=\boldsymbol{E}_{1}^{2}+\boldsymbol{E}_{2}^{2}+\cdots+\boldsymbol{E}_{q}^{2}
$$

$$
I_{\Sigma}=I_{1}+I_{2}+\cdots+I_{q} \text {. }
$$

Let us calculate the individual intensity terms - the intensities contributed by the individual groups of coherent rays. Let there be $t$ rays in a group $g=1 . q$. Here the phase of the incoming wave begins to play a role. In the case of arbitrary scenes, the vectors $\mathbf{E}_{g j}, j=1 . . t$ may have an arbitrary direction. Therefore, it does not make sense to remove the brackets, as in formula (2). In paper [40], a data structure was created to group rays arriving at a point from almost the same directions. Then the sum of pairwise interference terms within these groups was calculated. In this case, the interference of coherent rays coming from different directions could be lost. In paper [17], consideration was restricted to a case when interfering rays come from identical directions and have identical polarization planes, that is, formula (4) was used. In $[18,19]$ the calculations were based on the amplitude division algorithm [5] and formula (4).

In our approach, it is proposed first to directly calculate the field $\mathbf{E}_{g}$ of this group, and then calculate the intensity by simply squaring the amplitude, according to the definition. Recall that the $j$-th ray comes to point $P$ with a value of the vector $\boldsymbol{E}_{j}=\boldsymbol{E}_{j 0} e^{i \phi_{j}}$, where $\phi_{j}$ is the phase, which is calculated using the optical path passed by the ray according to the above algorithm. Then

$$
\boldsymbol{E}_{g}=\boldsymbol{E}_{10} e^{i \phi_{1}}+\boldsymbol{E}_{20} e^{i \phi_{2}}+\cdots+\boldsymbol{E}_{t 0} e^{i \phi_{t}} .
$$

Note that each term here is a vector in space. Figure 1 shows the vector of the resulting electric field at different scene points where the same three coherent rays meet: on the left 
for point $P_{1}: \boldsymbol{E}_{g}\left(P_{1}\right)=\boldsymbol{E}_{10} e^{i \phi_{1}\left(P_{1}\right)}+\boldsymbol{E}_{20} e^{i \phi_{2}\left(P_{1}\right)}+\boldsymbol{E}_{30} e^{i \phi_{3}\left(P_{1}\right)}$, and on the right for point $P_{2}: \boldsymbol{E}_{g}\left(P_{2}\right)=\boldsymbol{E}_{10} e^{i \phi_{1}\left(P_{2}\right)}+\boldsymbol{E}_{20} e^{i \phi_{2}\left(P_{2}\right)}+\boldsymbol{E}_{30} e^{i \phi_{3}\left(P_{2}\right)}$. For simplicity, a plane case is shown. The field vectors of the different rays are denoted by solid arrows of different colors, and the resulting vector is denoted by black color. An auxiliary construction for the addition of vectors is shown by dotted lines. The figure shows that the length of the resulting vector can vary significantly from point to point and, hence, the intensity is the squared length. Similar constructions can be found in books [11, 41] in a different context: constructing diffraction pictures.
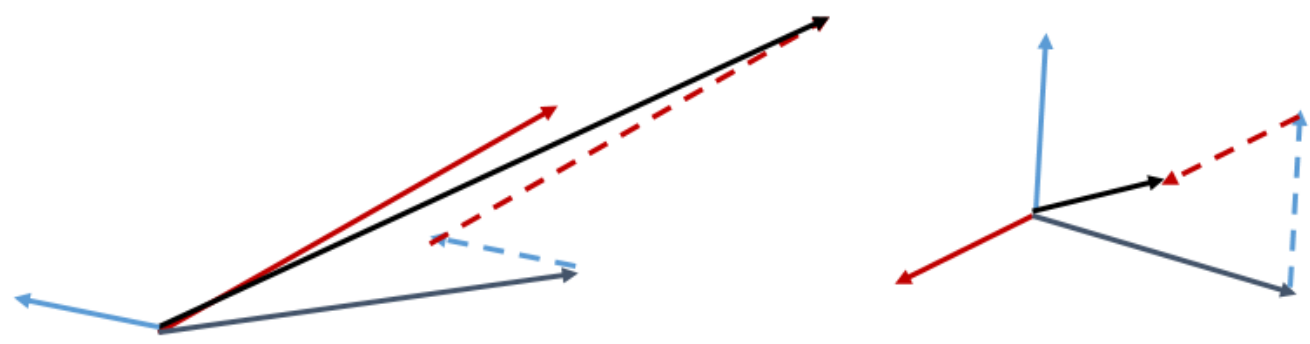

Fig. 1. Calculation of the resulting electric field vector (black) at two scene points.

\subsection{Phase jumps at reflection}

In order to correctly calculate the current phase, information about the optical path is not sufficient. It is also necessary to consider the jumpwise phase changes in the reflection of light at the interface $\Gamma$ between two transparent isotropic media with refractive indices $n_{1}$ and $n_{2}$. According to $[5,18]$, in reflection from the medium with the larger refractive index the phase shifts by $\pi$ :

$$
\phi_{\text {new }}=\phi_{\text {old }}-\pi, \text { if } n_{1}<n_{2}
$$

As a rule, this treatment is made when considering rays of unpolarized light. However, in what follows a subtler approach is considered. To describe the interaction of a ray with $\Gamma$, the Fresnel formulas are typically used (see $[5,6,20,30]$ ). For this, for an incident ray of even polarized light the electric field vector $\boldsymbol{E}$ in the general case is decomposed into two components: polarized perpendicularly to $\boldsymbol{E}_{\perp}$ and parallel to $\boldsymbol{E}_{\|}$of the incidence plane. The incidence plane is determined by the incident ray and the normal to the surface. In the case of normal incidence, when these vectors are collinear, no decomposition into the components is made.

Let a fully linear polarized ray with electric vector $\boldsymbol{E}$ strike the interface between two transparent media. We use the following notation: $\alpha$ - angle of incidence, $\gamma-$ angle of refraction, $\beta$ - Brewster's angle, $\operatorname{tg} \beta=n_{2} / n_{1}$. Let us decompose $\boldsymbol{E}$ into two components: $\boldsymbol{E}=\boldsymbol{E}_{\|}+\boldsymbol{E}_{\perp}$, one in the incidence plane and the other perpendicular to it. Similar decompositions are made for $\boldsymbol{E}_{r}=\boldsymbol{E}_{r \|}+\boldsymbol{E}_{r \perp}$ of the reflected wave and $\boldsymbol{E}_{t}=\boldsymbol{E}_{t \|}+\boldsymbol{E}_{t \perp}$ of the refracted wave. With the Fresnel formulas we find the following relations between the amplitudes of the incident, reflected, and refracted waves:

$$
\begin{gathered}
\frac{E_{r \perp}}{E_{\perp}}=-\frac{\sin (\alpha-\gamma)}{\sin (\alpha+\gamma)}, \quad \frac{E_{t \perp}}{E_{\perp}}=\frac{2 \cos a \sin \gamma}{\sin (\alpha+\gamma)}, \\
\frac{E_{r \|}}{E_{\|}}=\frac{\operatorname{tg}(\alpha-\gamma)}{\operatorname{tg}(\alpha+\gamma)}, \quad \frac{E_{t \|}}{E_{\|}}=\frac{2 \cos a \sin \gamma}{\sin (\alpha+\gamma) \cos (\alpha-\gamma)} .
\end{gathered}
$$


In the case of normal incidence, there is no decomposition into the components, and the formulas are transformed as follows:

$$
\frac{E_{r}}{E}=\frac{n_{1}-n_{2}}{n_{1}+n_{2}}, \frac{E_{t}}{E}=\frac{2 n_{1}}{n_{1}+n_{2}}
$$

Total internal reflection is a specially considered case. Here

$$
E_{r \|}=E_{\|}, E_{r \perp}=E_{\perp} \text {. }
$$

Taking into account the angle of incidence $\alpha$ and Brewster's angle $\beta$ at the boundary $\Gamma$, the phases of reflected and refracted rays are corrected according to the following rules:

- Under refraction, the incident and refracted rays are co-phased.

- $\boldsymbol{E}_{\perp}$ : under reflection, the phase changes to $-\pi$, if $n_{1}<n_{2}$.

- $\boldsymbol{E}_{\|}$: under reflection, the phase changes to $-\pi$, if

$$
n_{1}<n_{2} \wedge \alpha<\beta \vee n_{1}>n_{2} \wedge \alpha>\beta
$$

For the case of total internal reflection, more complex expressions for the phase jump are used [42]:

$$
\delta_{\|}=2 \operatorname{arctg}\left(\frac{\sqrt{\sin ^{2} \alpha-n_{21}^{2}}}{n_{21}^{2} \cos \alpha}\right)-\pi, \delta_{\perp}=2 \operatorname{arctg}\left(\frac{\sqrt{\sin ^{2} \alpha-n_{21}^{2}}}{\cos \alpha}\right)
$$

Once the Fresnel formulas are applied, the resulting generated rays are formed by adding their coordinate components.

\subsection{Total informational load of linear polarized light tracing ray}

When calculating images, rays are traced from their sources. Let us consider a linear polarized light ray with attributes

$$
R=\left\{P_{0}, \operatorname{dir}, \mathbf{X}, \mathbf{Y}, A \operatorname{tr}\right\}
$$

Here the mathematical ray is $\left\{P_{0}\right.$, dir $\}$ and the corresponding right-handed coordinate system is $\{\mathbf{X}, \mathbf{Y}, \mathbf{d i r}\}, P_{0}$ is the ray origin, dir is the direction, and $A t r$ is the set of attributes. The coherence matrix of this ray is related to this coordinate system, that is, its polarization and intensity are specified. The ray strikes the scene surface and generates new (reflected and refracted) rays. In this implementation, we take a coordinate system such that the electric vector oscillations are along the $\mathbf{X}$-axis. The following set of attributes is sufficient for calculating images taking into account interference:

- $\lambda$ - light wavelength. It was mentioned in Section 3.2 that only monochrome rays are used for spectral rendering.

- Light $_{i d}$ - identifier of the ray-generating point light source or of the differential area of the areal source. The program can assign unique identifiers (numeric or text ones). Let the zero value mean that a given ray on its further path will not be coherent with any other ray. Two rays are coherent if their source identifiers are nonzero and coincide.

- $S C_{\max }$ - limiting coherence length.

- $M_{h g}-2 \times 2$ coherence matrix. The intensity $I(\lambda)$ is calculated with the matrix trace.

- $O p$ - optical path from the source used to calculate the current phase of an electromagnetic wave.

- $\sum_{\delta}$ - phase jump accumulated under reflections.

The generated (reflected and refracted) rays inherit some of the attributes, or they are recalculated at the contact of the generating ray with the scene surface. 
To account for interference, the renderer can be modified as follows:

- Divide all rays coming to a screen point into mutual coherence groups.

- Determine the final phase and the electric field vector for each ray of each group.

- Obtain the resulting vector for each group.

- Add the squared lengths of the resulting vectors of the ray groups.

- Calculate the intensity.

Thus, all computational features of the interference accounting algorithm in a 3D scene with optically isotropic objects have been defined. Now let us consider numerical experiments illustrating the above statements.

\section{Interference in a scene with isotropic objects: experi- ments}

\subsection{Young's experiment}

Young's experiment is an experiment with wavefront division [5]. A parallel ray of monochromatic light strikes a screen $A$ with a small hole. Passing through the hole, the light reaches a second screen $B$ with two slits, $S_{1}$ and $S_{2}$, located at a distance $d$ from each other. The coherent rays coming from each of the slits interfere on a third screen, $C$ (image plane) located at a distance $l$ from $B$. Fig. 2 shows a scheme of the experiment.
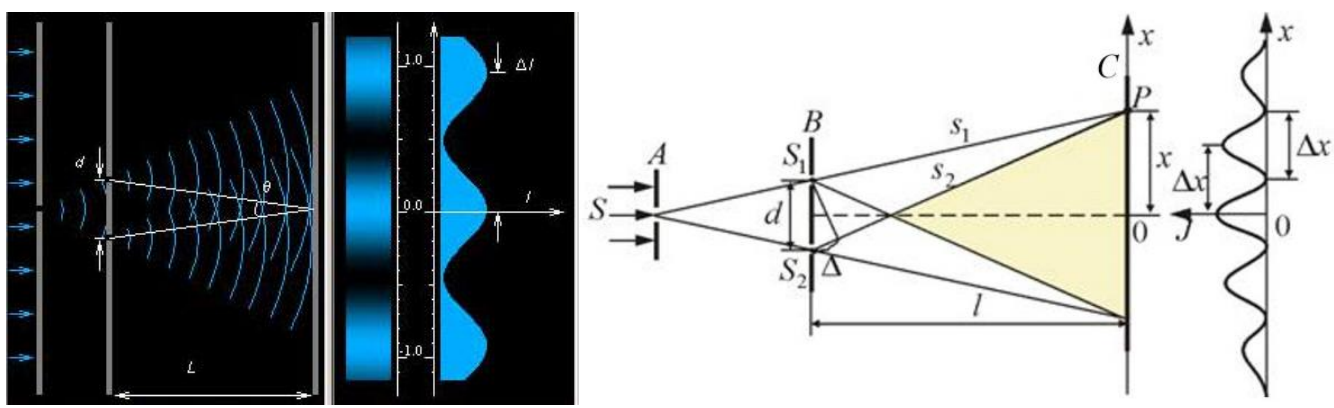

Fig. 2. Young's experiment, illustration from [43].

Let us construct a 3D scene for tracing the rays and calculating the image on the screen $C$ : a) monochrome coherent light sources $S_{1}$ and $S_{2}$ in the form of thin vertical parallel lines; b) screen $C$, on which the image is formed; $s_{1}$ and $s_{2}$ are the ray paths to point $P$. The refractive index of the medium is set equal to 1 and $\gg d$, as required in [5].

The right image in Fig. 3 is calculated by formula (4) with the following parameters: $\lambda=550 e^{-9} ; d=500 \cdot \lambda ; l=1000 \cdot d$.

In fact, in the scene the light rays do not cross any boundaries. Hence, the phase correction rules at reflections need not be applied.
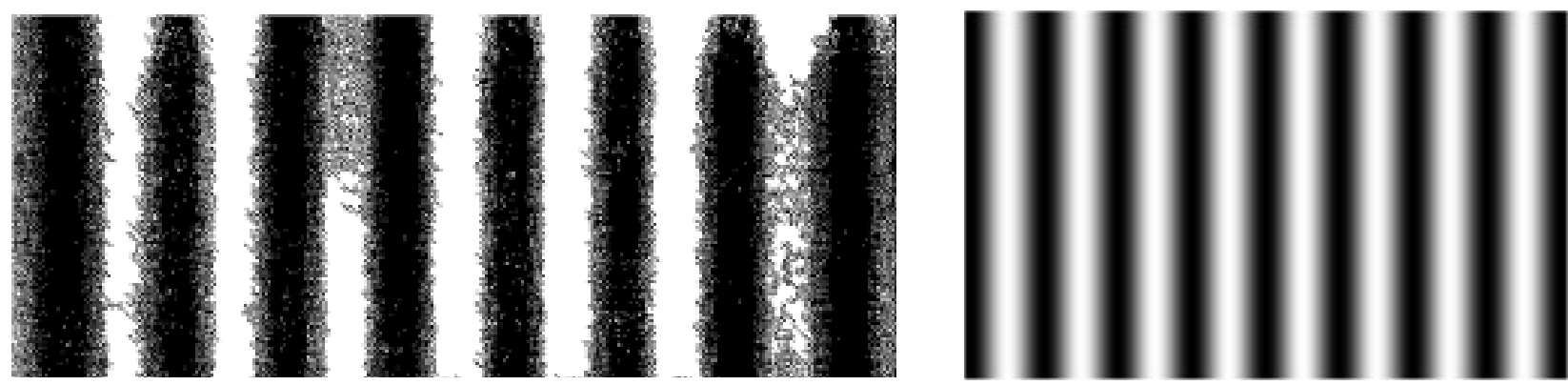
Fig. 3. Photo of Young's experiment from [5] (left), result of a numerical experiment from [28] (right).

\subsection{Amplitude division}

Another popular experiment demonstrating interference is that with amplitude division (the Fresnel method) [5]. In paper [28], a version of this experiment was simulated with the scene shown in Fig. 4 (left). A point source generates coherent monochrome rays of unpolarized light. A Shield prevents the rays from striking a screen (image), and the reflected ray $r_{0}$ is shielded by the Shield. Only rays interacting with a plane-parallel transparent plate fall on the screen. A target screen point $P$ is reached by two rays: $r_{1}$, the ray reflected from the upper boundary of the plate, and $r_{2}$, the ray reflected from its lower boundary. In other words, the interference of $r_{1}$ and $r_{2}$ is calculated; their amplitudes are obtained by dividing the amplitudes of different initial rays from the source. However, they are coherent, since there is only one source, and the resulting images are physically correct. In this experiment no additional devices are needed to bring the interfering rays to one screen point.

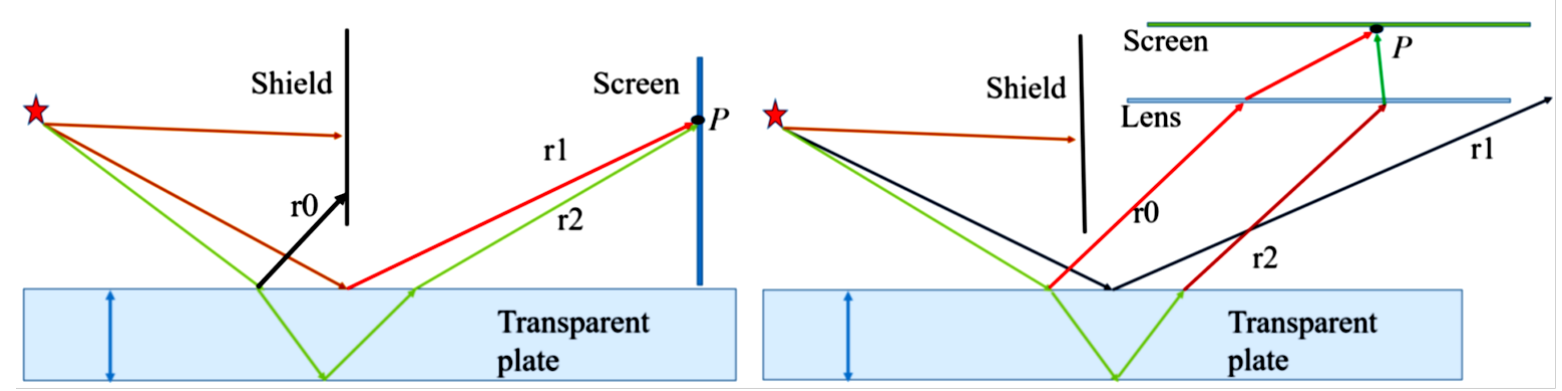

Fig. 4. Scene from [28] (left), scene corresponding to a popular description of the amplitude division experiment from [5] (right).

In the literature, this experiment (see [5] and others) is often described for the scene shown in Fig. 4 (right). To bring the interfering rays to one point, a lens is added whose focal plane is the recording screen. The ray $r_{1}$ used in [28] in this scene misses the lens and does not strike the screen.

Let us perform a series of experiments. Figure 5 shows an image calculated in spectral rendering by most of the existing renderers, since only the sum of intensities, $I_{1}+I_{2}$, coming with two rays (but not coherence) is taken into account. 


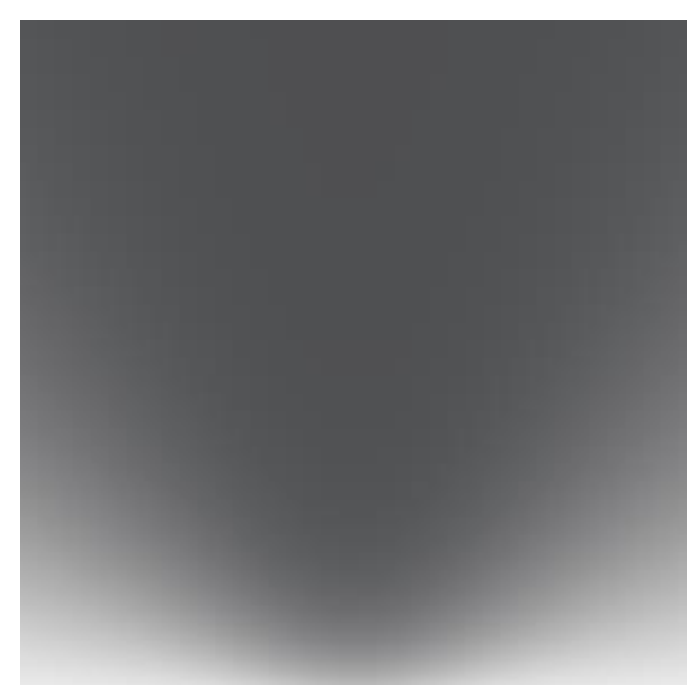

Fig. 5. Image calculation without coherence.

In Fig. 6 (left), coherence is taken into account, formula (4) is used, and the phase difference is calculated only on the basis of the difference of the optical paths. Let us take into account the phase jump when ray $r_{1}$ is reflected from a denser medium (Fig. 6, right). One can see that the picture has changed: the colored stripes seem to have shifted.
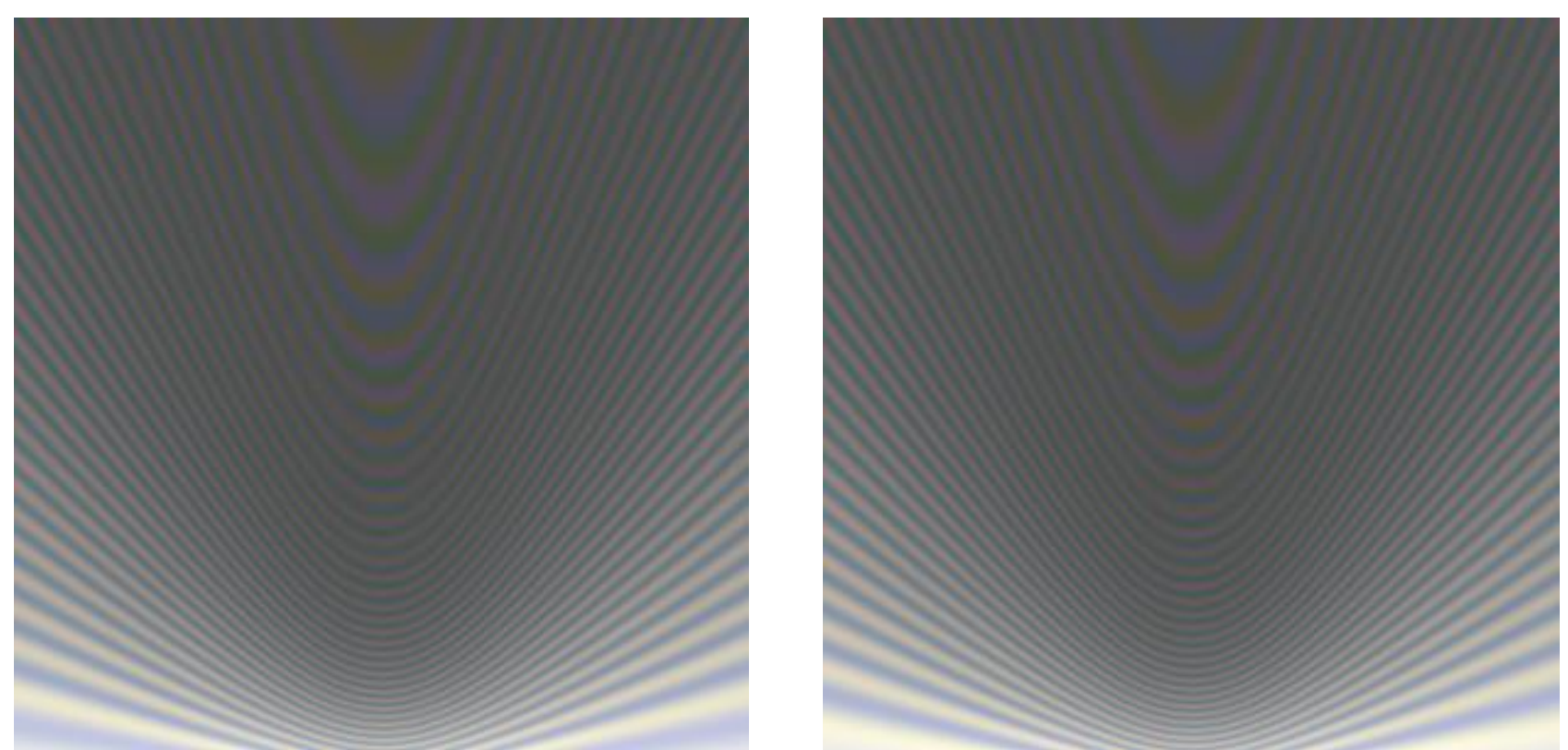

Fig. 6. Coherence and phase difference taken into account only on the basis of the difference in optical paths, calculation by formula (4) (left); calculation taking into account phase jump $\pi$ under reflection (right).

Let us calculate the image by the Fresnel formulas, with an unpolarized light ray represented as the sum of rays polarized parallel to the incidence plane and perpendicular to it, $R=R_{\|}+R_{\perp}$, and use formulas (8) and (9). According to the theory, the intensity at point $P$ is calculated by adding up two intensities, $I=I_{\|}+I_{\perp}$, see Fig. 7 (right).

The two images shown in Fig. 7 in an RGB format are as follows: the total number of pixels is 810000 ; the number of different pixels is 79041 ; the maximum difference $(\mathrm{r}+\mathrm{g}+\mathrm{b})=60$ at $(12,42)$; the maximum difference between the components $(\mathrm{r}|\mathrm{g}| \mathrm{b})$ is 31 at $(10,49)$. That is, the difference is an almost black image. It turns out that in some cases the coarser model in Fig. 7 (left) gives a rather good image. Fig. 8 shows histogram stretching to see places corresponding to the maximum discrepancy. This discrepancy is, most likely, due to 
the fact that in Fig. 7 ( left) all rays were processed according to the rules for rays with perpendicular polarization $R_{\perp}$, and the effect of Brewster's angle on $R_{\|}$was not taken into account (see formula (9)). Note that the dark "parabolic" stripe in Fig. 8 is located in the vicinity of Brewster's angle.
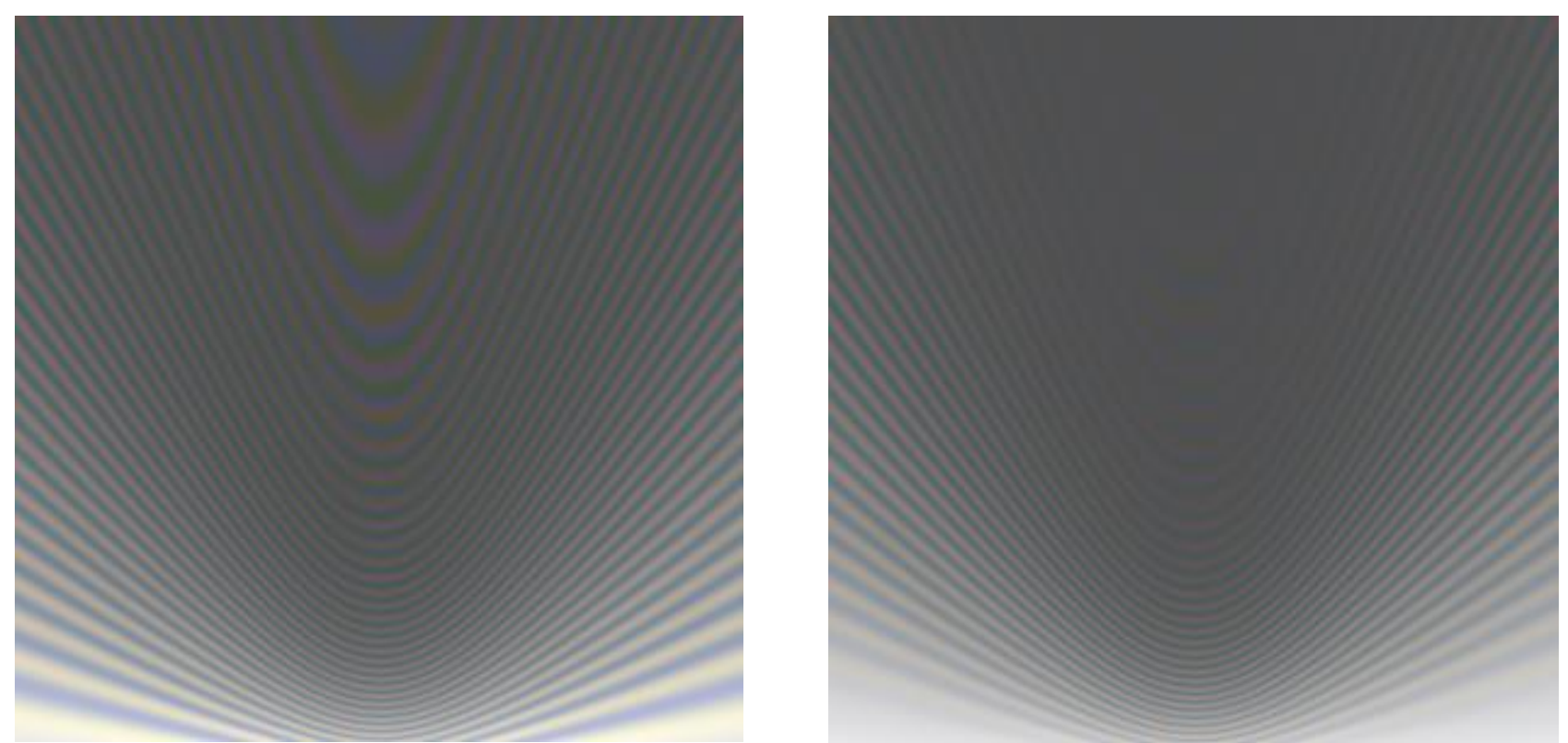

Fig. 7. Right image of Fig. 6 (left); image calculated on the basis of decomposition, $R=R_{\|}+R_{\perp}$ (right).

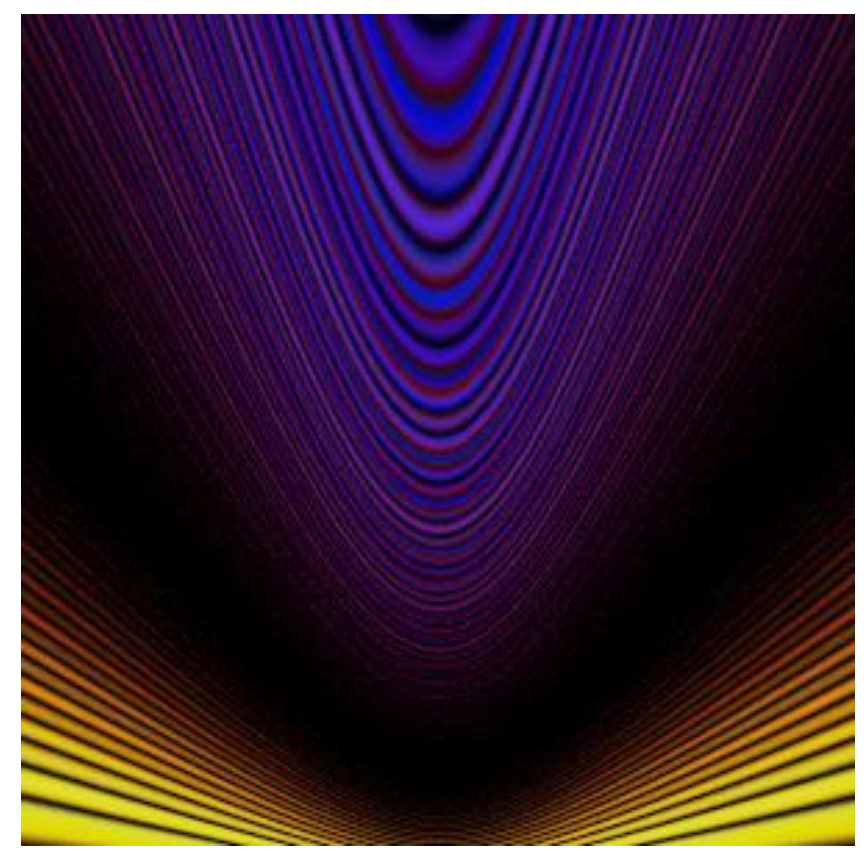

Fig. 8. Histogram stretching of the difference of images shown in Fig. 7.

\section{Conclusions}

In many experiments (as shown in the computer simulation of Young's experiment) and in the simulation of optical devices, the approach not taking into account Brewster's angle is quite sufficient (see the above images), especially when the incidence angles are mostly near zero or reflection is ignored. Our experiments have also shown that when using the Fresnel decomposition the perpendicular component makes a more significant contribution to the image than the parallel one. Note that the intensity of a ray from a point source decreases as the squared distance. This was not taken into account in the experiments, 
since major attention was given to how allowance for polarization or correct calculation of the phase shift affect the image of interference. The user can choose a suitable version of these methods.

A comparison of the images calculated with various physical correctness shows that any of them satisfies the requirements of photorealism in works aimed at obtaining a rich palette of rainbow colors.

Let us consider Fig. 4. All numerical experiments were performed for the left scene. These are model experiments, and the rays coming from a source to a point $P$ have been accurately calculated and, hence, their phases have also been accurately calculated. The problem is more complicated if we develop a renderer taking into account possible interference. The renderer calculates an image on some image plane consisting of pixels. Two or more coherent rays moving around the scene get into a certain pixel or subpixel. But a pixel is not a point! Nevertheless, a possible solution is to calculate interference for all coherent rays getting into a pixel. In our opinion, this problem was the reason for ignoring optical dispersion in the experiments reviewed in Section 2. In the introduction, an approach from [10] was discussed: tracing not with mathematical, but with "solid" rays. This solution would greatly increase the complexity of calculations for arbitrary scenes, and make accounting for interference practically unacceptable due to high computational complexity even in the near future, despite the fast development of computer technology. For the same reason, no algorithms based on tracing with a cone, pencil, etc. instead of a mathematical ray have been widely used in practice.

The right scene in Fig. 4 includes a lens which makes the calculation more difficult: optical dispersion, spherical aberration, etc. In fact, for this scene the problem as a whole must be solved. Eye observation is often simulated by using a system of lenses.

In conclusion, it should be noted that this problem calls for a separate study, which we plan to do in the nearest future.

This work was carried out under state contract with ICMMG SB RAS (0315-2019-0001).

\section{References}

1. Bondarev A. E. On visualization problems in a generalized computational experiment // Scientific Visualization, 2019, Vol. 11, № 2, pp. 156-162 (doi:10.26583/sv.11.2.12)

2. Debelov V. A., Kozlov D. S. Visualization of light polarization to debug ray tracing algorithms // Scientific Visualization, 2013, Vol. 5, № 5, p. 71-87.

3. Xiaoru Y., Minh N., Yingchun L., et al. Tsunami and Earthquake Visualization Inspired by Light $2006 . \quad$ Available at: https://www.researchgate.net/publication/228777380_Tsunami_and_Earthquake_V isualization_Inspired_by_Light_Interference. Accessed 20 Mar 2020.

4. Foley J. D. et al., Computer graphics, principles and practice, 2nd ed. Addison-Wesley, Reading, Mass., 1996.

5. Born M., Wolf E. Principles of Optics: Electromagnetic theory of propagation, interference and diffraction of light. Cambridge Univ. Press, 1980.

6. Landsber G. S. Optika [Optics], 6th ed. Moscow: FIZMATLIT, 2003 [in Russian].

7. Shaskolskaya M. P. Kristallografiya [Crystallography]. Moscow: Vysshaya Shkola, 1976 [in Russian].

8. Bloss D. An introduction to the methods of optical crystallography. NY: Holt, Rinehartand, and Winston, 1961.

9. Vasilyeva L. F., Debelov V. A., Shelepaev R. A. O razrabotke virtualnogo microskopa [On development of virtual microscope] // Proceedings of Intl. Conf. SCVRT2018, Puschino, TsarGrad, Russia, Novermber 20-23, 2018, pp. 325-332. [in Russian].

10. Harvey J. E., Irvin R. G., Pfisterer R. N. Modeling physical optics phenomena by complex ray tracing // Optical Engineering, Vol. 54, №. 3, 12 p., 2015, (DOI:10.1117/1.OE.54.3.035105) 
11. Nagibina I. M. Inerferentsiya i difraktsiya sveta [Inteference and diffraction of light], 2nd ed. Leningrad: Mashinostroenie, 1985. [in Russian].

12. Novitskii L. A. Optiko-elektronnye pribory dlya nauchnyh issledovaniy [Optoelectronic devices for scientific research]. Moscow: Mashinostroenie, 1986. [in Russian].

13. Devlin K., Chalmers A., Wilkie A., Purgathofer W. STAR: Tone reproduction and physically based spectral rendering // Eurographics 2002, pp. 101-123.

14. Maxwell render, 2013, http://www.nextlimit.com/maxwell/. Accessed 20 Mar 2020.

15. Dias M. L. Ray tracing interference color // IEEE Computer Graphics \& Applications, Vol. 11, № 2, pp. 54-60, 1991.

16. Glassner A. S. Andrew Glassner's Notebook Soap Bubbles: Part 2 // IEEE Computer Graphics and Applications, Vol. 20, № 6, pp. 99-109, 2000.

17. Gondek J. S., Meyer G. W., Newman J. G. Wavelength Dependent Reflection Functions // Computer Graphics (Proc. Siggraph'94), ACM Press, New York, pp. 213-219, 1994.

18. Sun Y., Fracchia F. D., Calvert T. W., Drew M. S. A spectrally based framework for realistic image synthesis // Visual Computer, Vol. 17, № 7, pp. 429-444, 2001.

19. Sun Y., Fracchia F. D., Calvert T. W., Drew M. S. Deriving Spectra from Colors and Rendering Light Interference // IEEE Computer Graphics and Applications, № 4, pp. 1-8, 1999.

20.Sun Y., Wang Q. Interference shaders of thin films // Comput. Graph. Forum, Vol. 27, Iss. 6, pp. 1607-1631, 2008.

21. Pixar's RenderMan, https://renderman.pixar.com/. Accessed 20 Mar 2020.

22. Iwasaki K. et al. Real-time rendering of soap bubbles taking into account light interference // Proceedings of Computer Graphics International Conference CGI, pp. 344 348, 2004.

23. Debelov V. A., Kozlov D. S. STAR: Rendering of transparent optically anisotropic objects // Conference Proceedings of Graphicon'2013, Vladivostok, Russia, pp. 50-55, 2013.

24. Guy S., Soler C. Graphics Gems Revisited // ACM Trans. Graphics, Vol. 23, № 3, pp. 231-238, 2004.

25. Weidlich A., Wilkie A. Realistic Rendering of Birefringency in Uniaxial Crystals // ACM Trans. Graphics, Vol. 27, № 1, pp. 6:1-6:12, 2008.

26. Debelov V. A., Kozlov D. S. A Local Model of Light Interaction with Transparent Crystalline Media. // IEEE Transactions on visualization and computer graphics, Vol. 19, № 8, pp. 1274-1287, 2013.

27. Kozlov D. S., Debelov V. A. Algorithm for photorealistic rendering of transparent optically active isotropic crystals // Programming and Computer Software, Vol. 41, № 5, pp. 267-272, 2015.

28. Debelov V. A. Interferenciya sveta, izotropnye prozrachnye ob'ekty, trassirovka luchey [Light interference, isotropic transparent objects, ray tracing] // Conference Proceedings of Graphicon'2015, pp. 168-173, 2015. [in Russian].

29. Vasilyeva L. F., Debelov V. A. Evolutsiya modeli lucha sveta dlya renderinga [Evolution of rendering light ray representation] // Proceed. of The International Scientific Conference of The Moscow Institute of Physics and Technology (State University) on Situational Centers and Information-Analytical System 4i Class for Monitoring and Security Tasks - SCVRT1516, November 21-24, 2015-2016, Puschino, TsarGrad, Russia, pp. 178-184. [in Russian].

30. Debelov V. A., Kushner K. G., Vasilyeva L. F. Lens for a Computer Model of a Polarizing Microscope // Mathematica Montisnigri, Vol. 41, pp. 151-165, 2018.

31. Cornell Box, http://www.graphics.cornell.edu/online/box, 1998. Accessed 20 Mar 2020. 
32. Debelov V. A., Kozlov D. S. Rendering of translucent objects, verification and validation of algorithms // Conference Proceedings of the WSCG'2012, Plzen, pp. 189-196, 2012.

33. Lindsay C., Agu E. Physically-based real-time diffraction using spherical harmonics // Lecture Notes in Computer Science, Vol. 4291, pp. 505-517, 2006.

34. Tandianus B., Johan H., Seah H. S. et al. Spectral caustic rendering of a homogeneous caustic object based on wavelength clustering and eye sensitivity // Visual Computer, Vol. 31, № 12, p. 1601-1614, 2015 (DOI: 10.1007/s00371-014-1037-z)

35. O’Neil E. L. Introduction to statistical optics. Addison-Wesley, 1963.

36. Tannenbaum D. C., Tannenbaum P., Wozny M. J. Polarization and birefringency considerations in rendering // Comp. Graphics (SIGGRAPH '94 Proceedings), ACM Press. pp. 221-222, 1994.

37. Whitted T. An Improved Illumination Model for Shaded Display // Comm. ACM, Vol. 23, № 6, pp. 343-349, 1980.

38.Glassner A.S. How to Derive a Spectrum from an RGB Triplet // IEEE Computer Graphics Applications, Vol. 9, № 4, pp. 95-99, 1989.

39. Methods to obtain coherent waves in optics, http://mashxxl.info/page/237202153160037004017084166207088218106096019211/. Accessed 20 Mar 2020.

40.Kozlov D. S. Algoritm renderinga po metodu Monte-Karlo petrograficheskih shlifov odnoosnyh kristallov [Algorithm for Monte Carlo rendering of cross sections of uniaxial crystalls] // Proseed. of Young Scientists School, Intl. Conf. Graphicon'2009, Moscow State University, 2009. [in Russian].

41. Ahmanov S. A., Nikitin S. Yu. Fizicheskaya optika [Physical optics], 2nd ed. Moscow State University, Nauka, 2004. [in Russian].

42. Kizel V. A. Otrazhenie sveta [Reflection of light]. Nauka, 1973. [in Russian].

43. Kuznetsov S. I. Klassicheskiy interferentsionnyy opyt Yunga [Young's classic interference experiment], http://9ooigr.net/prezentatsii/fizika/Interferentsija-voln/o19Klassicheskij-interferentsionnyj-opyt-JUnga.html. Accessed 20 Mar 2020. 